\title{
Retrospective diagnosis of SARS-CoV-2 infection in patients with Long COVID by measuring specific $T$ cell mediated IL-2 release.
}

\author{
Benjamin A. Krishna
}

University of Cambridge

Eleanor Y. Lim

University of Cambridge

\section{Lenette Mactavous}

Cambridge University Hospitals NHS Foundation Trust

\section{NIHR BioResource Team}

\section{Paul Lyons}

Cambridge Institute for Medical Research https://orcid.org/0000-0001-7035-8997

\section{Rainer Doffinger}

Cambridge University Hospitals NHS Foundation Trust

John Bradley

University of Cambridge https://orcid.org/0000-0002-7774-8805

Kenneth G. C. Smith

Univerisity of Cambridge

John Sinclair

Cambridge University

Nicholas J. Matheson

University of Cambridge https://orcid.org/0000-0002-3318-1851

Paul J. Lehner

University of Cambridge https://orcid.org/0000-0001-9383-1054

Mark R. Wills ( $\square$ mrw1004@cam.ac.uk)

University of Cambridge

Nyaradzai Sithole ( $\nabla$ ns519@cam.ac.uk)

University of Cambridge https://orcid.org/0000-0002-8020-223X

\section{Article}

Keywords: SARS-CoV-2, Long COVID, memory T cells

Posted Date: October 21st, 2021 
DOl: https://doi.org/10.21203/rs.3.rs-989434/v1

License: (c) (1) This work is licensed under a Creative Commons Attribution 4.0 International License. Read Full License 


\section{Abstract}

National Institute for Health and Care Excellence (NICE) guidelines define Long COVID as signs and symptoms that develop during or after an infection consistent with COVID-19, that continue for more than 12 weeks and are not explained by an alternative diagnosis. Long COVID is as yet poorly understood and difficult to diagnose. The diagnostic complexity of Long COVID is compounded in many patients who were infected with SARS-CoV-2 but not tested at acute presentation and are antibody negative. Given the diagnostic conundrum of Long COVID, we set out to design a SARS-CoV-2 specific T cell assay, to follow up a cohort of undifferentiated mostly non-hospitalised patients with Long COVID for up to 13 months. Here, we show that IL-2 release from SARS-CoV-2-specific memory T cells shows $>75 \%$ sensitivity and $>88 \%$ specificity in identifying individuals with confirmed SARS-CoV-2 infection $>6$ months after a positive PCR test.

\section{Introduction}

Since the initial reports of SARS-CoV-2 infection in December 2019, hundreds of millions of people have been infected, most of whom experience an asymptomatic or mildly symptomatic acute infection. However, follow up studies suggest that $0.2-30 \%$ of patients experience a plethora of persistent symptoms, variously termed 'Long COVID', 'post-acute sequelae of COVID-19' (PASC) or post-COVID syndrome [1-4]. This is consistent with previous coronavirus outbreaks where at least $10 \%$ of individuals infected with MERS (Middle east respiratory syndrome) or SARS-CoV-1 [5-7] experienced prolonged symptoms. However, unlike MERS and SARS-CoV-1 sequelae, Long COVID affects individuals irrespective of the severity of the acute illness. The remitting and relapsing nature of the illness, lack of consensus regarding the definition for Long COVID, heterogeneity of the disease and lack of biomarker/s makes the diagnosis of Long COVID challenging, with patients reporting a wide range of symptoms including fatigue, fever, headache, dyspnoea, and anosmia [8-10].

Diagnosis of SARS-CoV-2 infection using RT-qPCR detection of viral genome has sensitivity as low as $70 \%$ [11], ideally the test has to be performed during peak viral shedding, beyond which the rate of false negatives increases significantly [12]. This, combined with the lack of community RT-qPCR nasopharyngeal swab testing during the initial phase of the pandemic in 2020, means that a significant number of non-hospitalised patients presenting with symptoms consistent with Long COVID and are SARS-CoV-2 seronegative, are a significant diagnostic challenge, as there is no objective diagnostic record of past infection with SARS-CoV-2 [13]. Serological assays are useful but underestimate the prevalence of infection and immunity status to SARS-CoV-2 as antibodies wane over time; with mild illness, rapid viral clearance and low antibody response following acute infection, being the most common reasons attributed to failure to detect antibodies [14-21]. Furthermore, if serological assays that only measure anti-Spike antibodies are used for retrospective diagnosis, they are bound to be become less effective going forward with the introduction of SARS-CoV-2 vaccines that depend on Spike as the immunogenic antigen. 
Antigen-specific memory $\mathrm{T}$ cell responses are clinically useful for retrospective diagnosis of infection [22]. Interleukin-2 (IL-2) and Interferon (IFN)-g CD $4^{+}$and $\mathrm{CD} 8^{+} \mathrm{T}$ cell responses specific to SARS-CoV-2 spike $(\mathrm{S})$, nucleocapsid $(\mathrm{N})$ and membrane $(\mathrm{M})$ peptides are detected in PCR-positive individuals up to 12 months after infection, including in those who are seronegative [15, 23-30].

Given the limitations of serological assays and the confounding issue of vaccination in retrospective diagnosis of SARS-CoV-2, we decided to use a robust SARS-CoV-2 T cell based Fluorospot assay to measure IL-2 and IFN-g after stimulation with Spike (S) Nucleocapsid (N) and Membrane (M) peptides which to our knowledge has not been applied in the context of an undifferentiated cohort of Long COVID patients. Although other studies have used IFN-g for SARS-CoV-2 based T cell assays [31], we found that IL-2 was more sensitive than IFN-g for discriminating between a cohort of patients with RT-qPCR confirmed SARS-CoV-2 compared to an unexposed control group.

\section{Results}

\section{IL-2 release predicts prior SARS-CoV-2 infection in patients with Long COVID}

From May 2020 to August 2021, we recruited 72 patients who attended the Infectious Diseases led Long COVID clinic at Cambridge University Hospital (Addenbrooke's). The objective was to set up a T-cell based assay to retrospectively diagnose SARS-CoV-2 infection in patients with symptoms in keeping with Long COVID but were seronegative for both anti-Spike and anti-Nucleocapsid. In addition to the research bloods, routine clinical bloods, demographic and clinical data were collected. Of the 72 patients, 60/72 were non-hospitalised and 12/72 were hospitalised (Table 1) at the time of their initial illness. The median age of the patients was 46.5 years (interquartile range (IQR) 35-58 years) with $61.1 \%(44 / 72)$ female. Only $23.61 \%$ were SARS-CoV-2 antibody positive for both anti-Spike and anti-Nucleocapsid, whereas the majority, $76.39 \%$ were seronegative for both. None of the patients were positive for anti-Spike but not anti-Nucleocapsid or vice versa. Sixty-two and half percent had comorbidities, of which the most frequent was asthma and/or COPD (Table 1).

Of the 72 patients recruited, the salient symptoms associated with Long COVID were: fatigue (44\%, $32 / 72)$, shortness of breath $(8.3 \%, 6 / 72)$, brain fog/memory/concentration problems $(1.4 \%, 1 / 72)$, chest pains $(6.9 \%, 5 / 72)$, palpitations $(8.3 \%, 6 / 72)$ and only $(2.8 \%, 2 / 72)$ had persistent fever (Table 2$)$.

To address our objective, we set up a highly sensitive, dual-colour cytokine (IFN- $y$ and IL-2) T cell FluoroSpot assay to measure and characterise SARS-CoV-2 specific T cell responses to a pool of peptides generated according to the predicted amino acid sequence of Spike (S), Nucleocapsid $(\mathrm{N})$ and Membrane (M) proteins of the original Wuhan SARS-CoV-2 strain. We analysed IL-2 and IFN- $\gamma$ release from peripheral blood mononuclear cells (PBMCs) collected from the cohort of Long COVID patients.

For positive controls, we used PBMC samples from a cohort of patients with RT-qPCR proven SARS-CoV-2 infection (covering asymptomatic to severe disease). The latter group was stratified into samples taken 28 days (D28) and from the same patients 90 days (D90) and 180 days (D180) post diagnosis. This 
allowed us to assess the durability of IL-2 and IFN-g specific T-cell responses over time. We used PBMC samples from unexposed healthy blood donors collected between 2014-2018 as negative controls.

Intriguingly, median IL-2 responses were significantly higher (78.9\%-95.6\%) for all three SARS-CoV-2 peptide pools in our confirmed positive cohort at D28, D90, and D180 (Fig. 1a-d) relative to our unexposed negative control cohort. The overall percentage of the cohort who had IL-2 responses above the limit of detection (values below this limit were set at 0.1 to allow their visualisation on a logarithmic axis) for $\mathrm{S}, \mathrm{N}$ and $\mathrm{M}$ peptides was higher in every case for positive controls than for unexposed. We used an internal positive control comprised of a mixture of anti-CD3 antibody plus Staphylococcal enterotoxin B (SEB) to demonstrate that donor cells were capable of producing IL-2, and no difference noted in any group, albeit some reduction in D180 samples. Given that some studies have been carried out using IFN-g in SARSCoV-2 although in different settings, we determined the use of IFN-g in Long COVID but this was confounded by high background. Although the median IFN-g release T-cell responses to $\mathrm{S}, \mathrm{N}$ and $\mathrm{M}$ peptides increased in the RT-qPCR-confirmed SARS-CoV-2 cohort of subjects, at D28, D90 and D180 relative to unexposed control (Fig. 2a-d), differences were smaller than those seen for IL-2. Some PBMC from unexposed (pre-2019) donors had particularly high IFN-g responses to S, N and M which we attribute to T-cell cross-reactivity to circulating endemic human coronaviruses (Fig. 2a-d). Indeed, sequence analysis confirmed that regions of $\mathrm{S}, \mathrm{N}$ and $\mathrm{M}$ peptides are similar between SARS-CoV-2 and the endemic betacoronaviruses: HKU1 and OC43, (Fig. S1).

Given that our data reflect that IL-2 response show superiority over IFN-g as discriminator for past SARSCoV-2 infection, we therefore chose to develop our assay in Long COVID patients based on IL-2 response.

Although some of the Long COVID patients have now been vaccinated, we hereby only analysed data using samples taken prior to vaccination, to avoid confounding immune responses associated with vaccines. Based on the clinical history and temporal link, we expected that some patients were indeed infected with SARS-CoV-2, while others were likely to have been infected with other pathogens exhibiting overlapping symptoms with those of COVID-19. Due to this, we also stratified the Long COVID cohort into those who were seropositive for both anti-S and anti-N antibodies (17/72) and/or had a positive nasopharyngeal SARS-CoV-2 RT-qPCR swab (7/72), against those who were antibody negative and did not have a positive RT-qPCR test.

Our Long COVID cohort showed a range of IL-2 responses to S, M and N peptides, which is consistent with the cohort being comprised of some patients who genuinely had been infected and others who hadn't been infected with SARS-CoV-2. The analysis showed that all patients within the anti-S/anti-N seropositive group had detectable IL-2 T-cell responses to $S$ peptides, and all but one individual also responded to the $\mathrm{M}$ and $\mathrm{N}$ peptides (Fig. 3a-C). Overall, the Long COVID seropositive group was not statistically different from the D180 COVID-19 confirmed positive group. As expected, the Long COVID seronegative group was more varied, with some individuals having similar responses to $\mathrm{M}$ and $\mathrm{N}$ as our RT-qPCR COVID positive cohort, and others showing no detectable response (Fig. 3a-c). Taken together, our findings show that SARS-CoV-2 specific IL-2 responses (summarised in Table 3) are significantly 
stronger in the Long COVID cohort than in unexposed controls, which confers confidence to distinctly identify other patients likely to have been infected with SARS CoV-2 who lack a positive confirmatory SARS-CoV-2 RT-PCR or serological evidence of past infection.

\section{Combining T cell IL-2 responses to $\mathrm{N}$ and $\mathrm{M}$ allows for high sensitivity and specificity for past SARS-CoV- 2 infection}

Although SARS-CoV-2 specific T cell IL-2 responses to $\mathrm{S}, \mathrm{N}$, and $\mathrm{M}$ were on average stronger in confirmed anti-S/anti-N antibody positive Long-COVID patients than in unexposed donors, 25/56 unexposed donors had detectable responses to at least one peptide pool of $\mathrm{S}, \mathrm{N}$ or $\mathrm{M}$. This may represent cross reactivity to other coronavirus epitopes. We therefore compared responses across two pools, to screen out low-level cross reactivity and increase the degree of confidence to detect true positive, SARS-CoV-2 specific IL-2 responses.

We plotted individual patient and donor responses to each peptide pool and declared an individual positive if they had a response to $\mathrm{S} / \mathrm{M} / \mathrm{N}$ higher than any unexposed control, or if the individual responded to two or more ORFs with both signals above the upper quartile of the unexposed control samples. Responses above this background for more than one peptide pool were low for the unexposed control group (Fig. 4a-c), ranging from $11-15 \%$ of the total cohort, but much higher for RT-qPCR confirmed positive patients at D180 (Fig. 4d-f) at 73-81\% of the cohort. The seropositive patients with Long COVID showed similarly strong responses to multiple SARS-CoV-2 specific peptides, at $80-84 \%$ positive (Fig. $4 \mathrm{~g}$ i). Interestingly, $42-53 \%$ of Long COVID patients who were anti-S and anti-N seronegative showed clear positive T cell responses to two SARS-CoV-2 peptide pools (Fig. 4j-I). Therefore, this assay is highly sensitive for the retrospective diagnosis of SARS-CoV-2 infection, detecting $>73 \%$ of known positive samples, and will be an essential integral investigative tool for clinical diagnosis.

Given the increasing number of vaccinated individuals, we decided that using $\mathrm{N}$ and $\mathrm{M}$ responses to determine infection was the best approach to avoid confounding data from T cells induced by Spikebased vaccines. Indeed, samples from a selection of Long COVID patients after their first vaccine dose almost all showed increased IL-2 responses to spike (Fig. S2). Excluding spike as a marker of infection therefore avoids this problem. Using $N$ and $M$ responses exclusively, our test identified (28/38) $75 \%$ of known positives, at D180 post infection or (20/25) $80 \%$ of seropositive/RT-qPCR positive patients with Long COVID at least 6 months post infection (Fig. 5a). This compares favourably to antibody serology, where (33/38) of known positives, at D180 and (21/25) of seropositive/RT-qPCR positive patients with Long COVID were positive. Additionally, our assay revealed that $42.5 \%$ of the patients within the Long COVID cohort had strong virus-specific T cell evidence for past infection with SARS-CoV-2, despite being seronegative (Fig. 5a). Furthermore, of the 12 patients in our cohort who were hospitalised with COVID-19, $100 \%$ (12/12) were positive for SARS-CoV-2 IL-2 T cell responses while only 50\% (6/12) were antibody seropositive (Fig. 5b,c).

\section{Discussion}


Long COVID or 'post-acute sequelae of COVID-19' (PASC) is likely driven by multiple pathophysiological mechanisms with a resultant plethora of symptoms following infection with SARS-CoV-2. Lack of community testing during the initial phase of the pandemic in 2020, meant that many people who didn't meet the hospitalisation criteria, i.e., those who were asymptomatic to mildly symptomatic, were not tested by nasopharyngeal swab RT-qPCR or antigen tests. The lack of SARS-CoV-2 RT-qPCR and antigen tests has compounded the diagnostic dilemma in patients now presenting with Long COVID. As such, serum antibody levels are used to determine past infection. However, antibodies wane in up to $40 \%$ of patients beyond 6 months from the onset of symptoms, especially in those with asymptomatic or mild COVID-19 illness [15, 23, 32]. The failure of a significant number of people to sustain high levels of antibody production after infection was also evident in our Long COVID cohort, albeit the numbers were limited, nonetheless the data was consistent with known literature (Fig. S3). Going forward, this will prove to be a significant problem for retrospective diagnosis of SARS-CoV-2 infection and consequently diagnosing Long COVID. The loss of antibodies over time following coronaviruses closely related to SARS-CoV-2, i.e., SARS-CoV-1 and MERS infection also suggests that antibodies have considerable limitations especially when used in isolation as diagnostic tools for past SARS-CoV-2 infection [33]. The resultant diagnostic conundrum for those presenting with Long COVID has led to understandable frustrations for both clinicians and patients alike.

To address this urgent unmet clinical and scientific need, we tested whether a highly sensitive, dual-colour cytokine (IFN-y and IL-2) T cell FluoroSpot assay could determine which patients presenting with symptoms of Long COVID had evidence of past infection with SARS-CoV-2. We found that 180 days after a positive RT-qPCR result for SARS-CoV-2 infection, patients' T cells produced IL-2 in response to stimulation with Spike, Nucleocapsid and Membrane protein peptides at significantly higher levels than in unexposed control group from 2014-2018 (Fig. 1). This is consistent with published data that show decreasing antibody titres over time alongside detectable memory $T$ cell responses $[25,27]$.

Confirming the efficacy of our assay, patients diagnosed with Long COVID who were either antibody positive or who had a positive RT-qPCR result after nasopharyngeal swab were highly likely to respond to SARS-CoV-2 peptide stimulation with IL-2 release (Fig. 3). To improve specificity and stringency of our assay we excluded anti-Spike responses which could be caused by vaccination (Fig. S2) and used responses to $\mathrm{M}$ and $\mathrm{N}$ as criteria for a positive result (Fig. 4). Excluding Spike responses did not significantly reduce the number of identified patients, as most patients who responded to Spike also responded to both $\mathrm{N}$ and $\mathrm{M}$, consistent with the findings of others [29]. Using this approach, we were able to identify that $\sim 50 \%$ of seronegative patients with symptoms in keeping with Long COVID in our cohort, had indeed been infected with SARS-CoV-2 at some point in their illness trajectory (Fig. 5).

Our findings are consistent with other coronaviruses where cellular immunity is also important [34]. T cell responses were detectable $>10$ years after infection with SARS-CoV- 1 despite undetectable IgG in 2/23 patients [23, 35-38], suggesting that with the passage of time, $T$ cell-based assays such as our fluorospot approach are more effective and sensitive than antibody serology. There is also proof of concept in the use of T cell-based ELISpot assays for diagnosis of latent Mycobacterium Tuberculosis (using antigens 
ESAT-6 and CFP-10) [22]. Indeed, T-SPOT.COVID from Oxford Immunotec uses IFN- $\gamma$ release as a measure of past SARS-CoV-2 infection (https://www.tspotcovid.com/), which has been used as a measure of historical SARS-CoV-2 infection by some [39] and proposed as a diagnostic tool by others [31] but never investigated in the context of Long COVID. We concur with their analysis showing that $\mathrm{T}$ cell responses do not wane as quickly as antibody serology. However, our data suggest that IL-2 is a superior discriminator to IFN- $\gamma$, as the latter exhibits higher results in unexposed individuals (Fig. 1), hence the concern it could lead to higher false positive rates.

In our Long COVID cohort, based on serology test, 21/72 patients had been infected with SARS-CoV-2; however, using the T cell assay, interestingly and more importantly, this enabled us to identify an additional 20 patients, therefore, almost doubling the number of patients who had previously been infected with SARS-CoV-2. Based on our findings we propose that a T cell-based assay should be used in conjunction with antibody assays when diagnosing past SARS-CoV-2 infection. This assay would not be any more invasive than antibody serology as blood is used for both tests, and the T cell assay can be performed using only $10^{7}$ PBMCs, or around $10 \mathrm{ml}$ of blood. It would be interesting in future work with a larger cohort to determine whether there is any correlation between likelihood of past infection with certain symptoms to ideally narrow the range of symptoms associated with Long COVID. Other attempts so far have found that fatigue is associated with female gender, pre-existing lung disease, severity of acute illness and increased convalescent antibody titres [48]. Our data does not show correlation between any particular symptoms or resolution of symptoms with IL-2 responses.

The T cell reactivity noted with all 3 peptide pools (S/N/M) in the unexposed donors is probably due to cross-reactivity with other betacoronaviruses, which has been previously reported [24, 40-44], and confirmed here using basic sequence alignment (Fig. S1). Indeed, 3/58 unexposed donors had strong responses to $\mathrm{S}, \mathrm{N}$ and $\mathrm{M}$ which were too high to disregard as background production. We used pools of peptides to stimulate PBMCs which covered the entire length of the SARS-CoV-2 open reading frames for $\mathrm{S} / \mathrm{N} / \mathrm{M}$. There is scope to modify the pool of peptides used, in-order to further reduce the false positive rate. We will start by excluding peptides that we identified by sequence alignment of S/N/M from SARSCoV-2 with the circulating human coronaviruses and SARS-CoV-1 (Fig. S1). This is likely to be a key issue as the SARS-CoV-2 T cell reactive unexposed individuals within the negative control group were likely infected with one or more of the four human circulating endemic coronaviruses that are known to cause the common cold. It is less likely that our Long COVID cohort have been infected recently with other coronaviruses, as infections with all respiratory viruses dropped during 2020 due to non-pharmacological interventions including social distancing and wearing of face masks, directed against SARS-CoV-2 [36, 45]. This will however become an issue for retrospective diagnosis of SARS-CoV-2 infection using T cellbased assays as countries end restrictions on social interactions, which will likely see resurfacing and the spread of circulating endemic coronaviruses. Reducing cross reactivity will therefore be paramount to attain higher accuracy in the future. Use of peptides from non-structural proteins to detect $T$ cell responses to SARS-CoV-2 has been done in other settings $[29,46,47]$, however, these are less immunogenic. 
Although no single test is likely to be the panacea for diagnosis of this complex heterogeneous disease, the development of a diagnostic assay with higher sensitivity to past SARS-CoV-2 infections than antibody serology would be beneficial to both Long COVID patients and clinicians in planning future treatments. Our assay has revealed that a large number (30.6\%) of patients with symptoms suggestive of Long COVID from the initial phase of the pandemic who had been missed out by serological assays, have indeed been exposed to SARS-CoV-2. Based on our findings we propose that IL-2 production, in addition to antibody assays, will allow for more sensitive detection of past SARS-CoV-2 infections. The assay can potentially be adapted to a simpler whole blood peptide stimulation assay with IL-2 ELISA readout, thereby, attain high-throughput advantages and easier to implement as a clinical diagnostic assay.

In conclusion, our study reveals a novel assay for retrospective diagnosis of SARS-CoV-2 infections that we hope will be adapted in the near future for use in clinical diagnostic laboratories. These results demonstrate the increased sensitivity of the SARS-CoV-2 T cell assay which should be used in conjunction with antibody serology for retrospective diagnosis of SARS-CoV-2 infection and longevity SARS-CoV-2 immunity studies.

\section{Methods}

Ethics and sample collection. Study participants were recruited between $31^{\text {st }}$ of May 2020 and $31^{\text {st }}$ of July 2021 from patients attending the Infectious Diseases led Long COVID clinic at Addenbrooke's Hospital. The majority were non-hospitalised patients from the initial phase of the pandemic and the clinical and epidemiological history played the most significant part in triaging patients into the Long COVID clinic. However, a combination of any of the following parameters were used to triage patients into the clinic; epidemiological and clinical history (both initially assessed by referring General Practitioners), a confirmed diagnosis of COVID-19 by nucleic acid amplification test (including point-of-care testing) and SARS-CoV-2 seropositivity. The Long COVID study patients were recruited and consented under the Cambridge COVID-19 NIHR BioResource joint Consent Form (Research Ethics Committee (NRES number (REC)) no. T1gC1) study NBR87.

The COVID confirmed hospitalised patients (Day28 \& Day90), were enrolled following admission to Addenbrooke's hospital, Royal Papworth and Cambridge and Peterborough Foundation Trust with a confirmed diagnosis of COVID-19 via a positive RT-qPCR test for SARS-CoV-2 as stated in [48].

Recruitment of inpatients at Addenbrooke's Hospital and health-care workers was undertaken by the National Institute for Health Research (NIHR) Cambridge Clinical Research Facility outreach team and the NIHR BioResource research nurse team as stated in [48]. Informed consent was obtained from all participants. Each participant provided $32 \mathrm{ml}$ of peripheral venous blood collected into a 9 -ml sodium citrate tube. Clinical data was collected at clinic visit and routine laboratory tests and inflammatory cytokine panel were assayed appropriately where clinically relevant.

\section{Serology testing}


SARS-CoV-2 serology by multiplex particle-based flow cytometry (Luminex): Recombinant SARS-CoV-2 N, $S$ and RBD were covalently coupled to distinct carboxylated bead sets (Luminex; Netherlands) to form a 3-plex assay. The S protein construct used is S-R/PP [49]. The RBD protein construct used is described by Stadlbauer et. al, [50]. Beads were first activated with 1-ethyl-3-[3-dimethylaminopropyl]carbodiimide hydrochloride (Thermo Fisher Scientific) in the presence of N-hydroxysuccinimide (Thermo Fisher Scientific), according to the manufacturer's instructions, to form amine-reactive intermediates. The activated bead sets were incubated with the corresponding proteins at a concentration of $50 \mu \mathrm{g} / \mathrm{ml}$ in the reaction mixture for 3 hours at room temperature on a rotator. Beads were washed and stored in a blocking buffer (10 mM PBS, 1\% BSA, 0.05\% NaN3).

The N-, S- and RBD-coupled bead sets were incubated with proband sera at a 1/100 dilution for $1 \mathrm{~h}$ in 96 well filter plates (MultiScreen HTS; Millipore) at room temperature in the dark on a horizontal shaker. Fluids were aspirated with a vacuum manifold and beads were washed three times with $10 \mathrm{mM}$ PBS/0.05\% Tween 20. Beads were incubated for $30 \mathrm{~min}$ with a PE-labelled anti-human IgG-Fc antibody (Leinco/Biotrend), washed as described above, and resuspended in $100 \mu \mathrm{l} \mathrm{PBS/Tween.} \mathrm{They} \mathrm{were} \mathrm{then}$ analysed on a Luminex analyser (Luminex / R\&D Systems) using Exponent Software V31. Specific binding was reported as mean fluorescence intensities (MFI). N protein was kindly provided by Dr Leo James. RBD was provided by Dr James Nathan. Trimeric S was provided by Dr John Briggs.

\section{PBMC isolation from patient blood}

Peripheral blood mononuclear cells (PBMCs) were isolated from citrated blood samples by layering blood onto Lymphoprep (Axis-shield, Oslo, Norway) and performing density gradient centrifugation at $1200 \mathrm{xg}$ for 10 mins. PBMCs at the interface were collected and washed $2 x$ in PBS.

\section{Dual FluoroSpot Assays}

We used peptide pools as recently published [51]: "A peptide pool was generated using the following: 1 . PepTivator SARS-CoV-2 Prot_S containing the sequence domains aa 304-338, 421- 475, 492-519, 683$707,741-770,785-802$, and 885 - 1273 and S1 N-terminal S1 domain of the surface glycoprotein ("S") of SARS-Coronavirus 2 (GenBank MN908947.3, Protein QHD43416.1). 2. The PepTivator SARS-CoV-2 Prot_S1 containing the aa sequence 1-692. The peptides used are $15 \mathrm{aa}$ amino acids with 11 amino acid overlaps."

$2 \times 10^{5}$ PBMCs suspended in TexMACS (Miltenyi Biotech) supplemented with $5 \%$ Human AB serum (Sigma Aldrich) were incubated on FluoroSpot plates coated with Human IFN- $\gamma$ and IL-2 antibodies [FluoroSpot (Mabtech AB, Nacka Strand, Sweden)] in duplicate with ORF mix peptides (final peptide concentration $2 \mu \mathrm{g} / \mathrm{ml} /$ peptide) as well as a TexMACS-only negative control and positive control mix [containing anti-CD3 (Mabtech AB), Staphylococcus Enterotoxin B, and Lipopolysaccharide (all SigmaAldrich)] at $37^{\circ} \mathrm{C}$ in a humidified $\mathrm{CO} 2$ atmosphere for $48 \mathrm{~h}$. The cells and medium were decanted from the plate and the assay developed following the manufacturer's instructions. Developed plates were read using an AID iSpot reader (Oxford Biosystems, Oxford, UK) and counted using AID EliSpot v7 software 
(Autoimmun Diagnostika GmbH, Strasberg, Germany) using distinct counting protocols for IFN-y and IL-2 secretion. Donor results were discounted from further analysis if there was less than $100 \mathrm{sfu}$ in the positive control relative to the background sfu. All data were then corrected for background cytokine production.

\section{Sequence analysis}

Sequences for S/N/M from SARS-CoV-2, SARS-CoV-1, OC43, HKU1, NL63 and 229E (GenBank numbers: QHD43416.1, NP_828851.1, YP_009555241.1, AGW27872.1, APF29063.1, NP_073551.1; QHD43423.2, AYV99827.1, AXX83383.1, ABG77571.1, ABI20791.1, AAA45463.1; QHD43419.1, ACZ71786.1, AAA45462.1, AGW27884.1, ABD34826.1, AAA45461.1) were aligned using ClustalX software [52]. Sequence alignments were visualised using UGene software [53] coloured to indicate areas of high percentage identity.

\section{Data Handling}

Data were determined to be non-parametric by Shapiro-Wilk analysis. We therefore used non-parametric statistical analysis (Mann-Whitney U, Kruskal-Wallis one-way analysis of variance, Wilcoxon signedrank test) throughout.

\section{Declarations}

\section{Acknowledgements}

We would like to thank the staff and patients at CUH NHS Foundation Trust. This work was funded by the Addenbrooke's Charitable Trust ( 900276 to NS) and supported by the NIHR Cambridge Biomedical Research Centre. NJM is supported by the MRC (TSF MR/T032413/1) and NHSBT (WPA15-02). PJL is supported by the Wellcome Trust (PRF 210688/Z/18/Z). The views expressed are those of the authors and not necessarily those of the NIHR or the Department of Health and Social Care. The funders had no role in the design, execution, or analysis of the study.

\section{Author Contributions}

Conceptualisation, MRW, NS; Methodology, MRW, NS, BAK; Investigation \& Data Collection, BAK, EYL, LM, RD, MRW, NS; Supervision - NJM, PJL, MRW, NS; Writing - Original Draft, BAK, MRW, NS; Writing Review \& Editing, BAK, EYL, LM, PL, RD, JB, KGCS, JS, NJM, PJL, MRW and NS.

\section{Competing Interests}

The authors declare no competing interests

\section{NIHR BioResource}


John Allison, Heather Biggs, John Bradley, Helen Butcher, Daniela Caputo, Matt Chandler, Debbie Clapham-Riley, Patrick Chinnery, Anne-Maree Dean, Eleanor Dewhurst, Christian Fernandez, Anita Furlong, Anne George, Barbara Graves, Jennifer Gray, Sabine Hein, Tasmin Ivers, Mary Kasanicki, Nathalie Kingston, Emma Le Gresley, Rachel Linger, Sarah Meloy, Alexei Moulton, Francesca Muldoon, Nigel Ovington, Roxana Paraschiv, Sofia Papadia, Isabel Phelan, Christopher Penkett, Venkatesh Ranganath, Jennifer Sambrook, Katherine Schon, Hannah Stark, Kathleen E Stirrups, Paul Townsend, Julie von Ziegenweidt, Neil Walker, Jennifer Webster

\section{References}

1. Statistics, O.f.N., The prevalence of long COVID symptoms and COVID-19 complications. 2020.

2. Thompson, E.J., et al., Risk factors for long COVID: analyses of 10 longitudinal studies and electronic health records in the UK. medRxiv, 2021.

3. Logue, J.K., et al., Sequelae in adults at 6 months after COVID-19 infection. JAMA network open, 2021. 4(2): p. e210830-e210830.

4. Petersen, M.S., et al., Long COVID in the Faroe Islands-a longitudinal study among nonhospitalized patients. Clinical infectious diseases: an official publication of the Infectious Diseases Society of America, 2020.

5. Hickie, l., et al., Post-infective and chronic fatigue syndromes precipitated by viral and non-viral pathogens: prospective cohort study. BMJ, 2006. 333(7568): p. 575.

6. Moldofsky, H. and J. Patcai, Chronic widespread musculoskeletal pain, fatigue, depression and disordered sleep in chronic post-SARS syndrome; a case-controlled study. BMC neurology, 2011. 11: p. 3737.

7. Ahmed, H., et al., Long-term clinical outcomes in survivors of severe acute respiratory syndrome and Middle East respiratory syndrome coronavirus outbreaks after hospitalisation or ICU admission: A systematic review and meta-analysis. J Rehabil Med, 2020. 52(5): p. jrm00063.

8. Sudre, C.H., et al., Attributes and predictors of long COVID. Nature medicine, 2021. 27(4): p. 626631.

9. Nabavi, N., Long covid: How to define it and how to manage it. 2020, British Medical Journal Publishing Group.

10. Nalbandian, A., et al., Post-acute COVID-19 syndrome. Nature medicine, 2021. 27(4): p. 601-615.

11. Woloshin, S., N. Patel, and A.S. Kesselheim, False Negative Tests for SARS-CoV-2 Infection Challenges and Implications. New England Journal of Medicine, 2020. 383(6): p. e38. 
12. La Marca, A., et al., Testing for SARS-CoV-2 (COVID-19): a systematic review and clinical guide to molecular and serological in-vitro diagnostic assays. Reproductive biomedicine online, 2020.

13. Ameratunga, R., et al., Perspective: diagnostic laboratories should urgently develop $T$ cell assays for SARS-CoV-2 infection. Expert Review of Clinical Immunology, 2021. 17(5): p. 421-430.

14. Long, Q.-X., et al., Clinical and immunological assessment of asymptomatic SARS-CoV-2 infections. Nature Medicine, 2020. 26(8): p. 1200-1204.

15. Ibarrondo, F.J., et al., Rapid decay of anti-SARS-CoV-2 antibodies in persons with mild Covid-19. New England Journal of Medicine, 2020. 383(11): p. 1085-1087.

16. Fergie, J. and A. Srivastava, Immunity to SARS-CoV-2: Lessons Learned. Frontiers in immunology, 2021. 12: p. 654165-654165.

17. Tan, W., et al., Viral Kinetics and Antibody Responses in Patients with COVID-19. medRxiv, 2020: p. 2020.03.24.20042382.

18. Bonifacius, A., et al., COVID-19 immune signatures reveal stable antiviral $T$ cell function despite declining humoral responses. Immunity, 2021. 54(2): p. 340-354.e6.

19. Wu, F., et al., Neutralizing antibody responses to SARS-CoV-2 in a COVID-19 recovered patient cohort and their implications. medRxiv, 2020: p. 2020.03.30.20047365.

20. Cox, R.J. and K.A. Brokstad, Not just antibodies: B cells and T cells mediate immunity to COVID-19. Nature Reviews Immunology, 2020. 20(10): p. 581-582.

21. Seow, J., et al., Longitudinal observation and decline of neutralizing antibody responses in the three months following SARS-CoV-2 infection in humans. Nature microbiology, 2020. 5(12): p. 1598-1607.

22. Pai, M., et al., Gamma interferon release assays for detection of Mycobacterium tuberculosis infection. Clinical microbiology reviews, 2014. 27(1): p. 3-20.

23. Tan, W., et al., Viral kinetics and antibody responses in patients with COVID-19. MedRxiv, 2020.

24. Sekine, $T$., et al., Robust $T$ cell immunity in convalescent individuals with asymptomatic or mild COVID-19. Cell, 2020. 183(1): p. 158-168. e14.

25. Sherina, N., et al., Persistence of SARS-CoV-2-specific $B$ and T cell responses in convalescent COVID-19 patients 6-8 months after the infection. Med (N Y), 2021. 2(3): p. 281-295.e4.

26. Grifoni, A., et al., Targets of T cell responses to SARS-CoV-2 coronavirus in humans with COVID-19 disease and unexposed individuals. Cell, 2020. 181(7): p. 1489-1501. e15. 
27. Scurr, M.J., et al., Whole blood-based measurement of SARS-CoV-2-specific T cell responses reveals asymptomatic infection and vaccine efficacy in healthy subjects and patients with solid organ cancers. medRxiv, 2021: p. 2021.06.02.21258218.

28. Zuo, J., et al., Robust SARS-CoV-2-specific T cell immunity is maintained at 6 months following primary infection. Nature immunology, 2021. 22(5): p. 620-626.

29. Peng, Y., et al., Broad and strong memory CD4+ and CD8+ T cells induced by SARS-CoV-2 in UK convalescent individuals following COVID-19. Nature immunology, 2020. 21(11): p. 1336-1345.

30. Hou, H., et al., Immunological memory to SARS-CoV-2 in convalescent COVID-19 patients at oneyear post-infection. Journal of Allergy and Clinical Immunology, 2021.

31. Petrone, L., et al., A whole blood test to measure SARS-CoV-2-specific response in COVID-19 patients. Clinical Microbiology and Infection, 2021. 27(2): p. 286. e7-286. e13.

32. Fergie, J. and A. Srivastava, Immunity to SARS-CoV-2: Lessons Learned. Frontiers in Immunology, 2021. 12: p. 716.

33. Tang, F., et al., Lack of peripheral memory B cell responses in recovered patients with severe acute respiratory syndrome: a six-year follow-up study. The Journal of Immunology, 2011. 186(12): p. 72647268.

34. Liu, W.J., et al., T-cell immunity of SARS-CoV: Implications for vaccine development against MERSCoV. Antiviral research, 2017. 137: p. 82-92.

35. Li, C.K.-f., et al., T cell responses to whole SARS coronavirus in humans. The Journal of Immunology, 2008. 181(8): p. 5490-5500.

36. Oh, H.-L.J., et al., Engineering $T$ cells specific for a dominant severe acute respiratory syndrome coronavirus CD8 T cell epitope. Journal of virology, 2011. 85(20): p. 10464-10471.

37. Da Guan, W., et al., Characteristics of traveler with Middle East respiratory syndrome, China, 2015. Emerging infectious diseases, 2015. 21(12): p. 2278.

38. Ng, O.-W., et al., Memory T cell responses targeting the SARS coronavirus persist up to 11 years post-infection. Vaccine, 2016. 34(17): p. 2008-2014.

39. Scherlinger, M., et al., Refining "Long-COVID" by a Prospective Multimodal Evaluation of Patients with Long-Term Symptoms Attributed to SARS-CoV-2 Infection. Infectious Diseases and Therapy, 2021: p. $1-17$.

40. Bacher, P., et al., Low-avidity CD4+T cell responses to SARS-CoV-2 in unexposed individuals and humans with severe COVID-19. Immunity, 2020. 53(6): p. 1258-1271. e5. 
41. Doshi, P., Covid-19: Do many people have pre-existing immunity? Bmj, 2020. 370.

42. Steiner, S., et al., HCoV-and SARS-CoV-2 cross-reactive T cells in CVID patients. Frontiers in immunology, 2020. 11.

43. Mateus, J., et al., Selective and cross-reactive SARS-CoV-2 T cell epitopes in unexposed humans. Science, 2020. 370(6512): p. 89-94.

44. Lipsitch, M., et al., Cross-reactive memory T cells and herd immunity to SARS-CoV-2. Nature Reviews Immunology, 2020. 20(11): p. 709-713.

45. Groves, H., et al., The impact of the COVID-19 pandemic on influenza, respiratory syncytial virus, and other seasonal respiratory virus circulation in Canada. medRxiv, 2021: p. 2021.04.15.21255591.

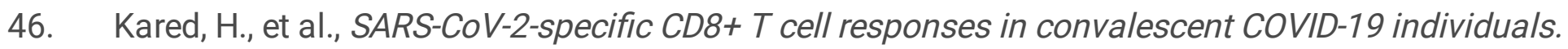
The Journal of clinical investigation, 2021. 131(5): p. e145476.

47. Takagi, A. and M. Matsui, Identification of HLA-A*02:01-restricted candidate epitopes derived from the non-structural polyprotein 1a of SARS-CoV-2 that may be natural targets of CD8(+) T cell recognition in vivo. Journal of virology, 2020. 95(5): p. e01837-20.

48. Bergamaschi, L., et al., Longitudinal analysis reveals that delayed bystander CD8+ T cell activation and early immune pathology distinguish severe COVID-19 from mild disease. Immunity, 2021. 54(6): $p$. 1257-1275.e8.

49. Xiong, X., et al., A thermostable, closed SARS-CoV-2 spike protein trimer. Nature structural \& molecular biology, 2020. 27(10): p. 934-941.

50. Stadlbauer, D., et al., SARS-CoV-2 seroconversion in humans: a detailed protocol for a serological assay, antigen production, and test setup. Current protocols in microbiology, 2020. 57(1): p. e100.

51. Collier, D.A., et al., Age-related immune response heterogeneity to SARS-CoV-2 vaccine BNT162b2. Nature, 2021: p. 1-9.

52. Thompson, J.D., T.J. Gibson, and D.G. Higgins, Multiple sequence alignment using ClustalW and ClustalX. Current protocols in bioinformatics, 2003(1): p. 2.3. 1-2.3. 22.

53. Okonechnikov, K., et al., Unipro UGENE: a unified bioinformatics toolkit. Bioinformatics, 2012. 28(8): p. 1166-1167.

\section{Tables}

Table 1: Demographic and baseline characteristics of the undifferentiated Long COVID patients 


\begin{tabular}{|c|c|}
\hline $\mathrm{n}$ & 72 \\
\hline \multirow[t]{2}{*}{ Age, median (IQR) } & 46.5 \\
\hline & $(35-58)$ \\
\hline $18-30$ years & 10 \\
\hline $31-45$ years & 25 \\
\hline $46-60$ years & 20 \\
\hline$>60$ years & 17 \\
\hline$\%$ male & $38.9 \%$ \\
\hline \%PCR positive & $9.72 \%$ \\
\hline$\%$ seropositive (both anti-S and anti-N) & $23.61 \%$ \\
\hline$\%$ seronegative & $76.39 \%$ \\
\hline \%hospitalised & $16.7 \%$ \\
\hline \%mild illness & $61.1 \%$ \\
\hline Comorbidities & $45 / 72$ \\
\hline Hypertension & $9 / 72$ \\
\hline Diabetes Mellitus & $4 / 72$ \\
\hline COPD, Asthma & $19 / 72$ \\
\hline Anxiety/Depression & $7 / 72$ \\
\hline Cancer/Immunosuppression & $3 / 72$ \\
\hline Obesity & $1 / 72$ \\
\hline Chronic Heart Disease & $3 / 72$ \\
\hline Time since symptoms (months +/- SD) & 6.97 \\
\hline & $+/-2.83$ \\
\hline
\end{tabular}

Table 2: Main symptoms at presentation of the Long COVID patients 


\begin{tabular}{|llllll|}
\hline Parameter/s & All & 18-30yrs & 31-45yrs & 46-60yrs & >60yrs \\
\hline Symptoms & & & & & \\
\hline Fatigue & 32 & 5 & 9 & 13 & 5 \\
\hline Shortness of breath & 6 & 1 & 3 & 1 & 1 \\
\hline Brain fog/memory/concentration problems & 1 & 0 & 0 & 1 & 0 \\
\hline Peripheral numbness & 1 & 0 & 1 & 0 & 0 \\
\hline Chest pains & 5 & 1 & 3 & 1 & 0 \\
\hline Palpitations & 6 & 2 & 2 & 2 & 0 \\
\hline Fever & 2 & 0 & 0 & 2 & 0 \\
\hline Altered taste/smell & 2 & 0 & 1 & 1 & 0 \\
\hline
\end{tabular}

Table 3: SARS-CoV-2 serology and IL-2 status of Long COVID patients

\begin{tabular}{|llllll|}
\hline & $\begin{array}{l}\text { All Long } \\
\text { CoVID } \\
\text { patients }\end{array}$ & $\begin{array}{l}\text { Non- } \\
\text { hospitalised }\end{array}$ & Hospitalised & $\begin{array}{l}\text { SARS-CoV-2 } \\
\text { antibody positive } \\
\text { (negative) }\end{array}$ & $\begin{array}{l}\text { IL-2 positivity (of } \\
\text { which } \\
\text { seronegative) }\end{array}$ \\
\hline $\begin{array}{l}\text { Age } \\
\text { categories }\end{array}$ & & & & & \\
\hline $18-30$ & 10 & 9 & 1 & $5(5)$ & $5(1)$ \\
\hline $31-45$ & 25 & 25 & 0 & $4(21)$ & $11(9)$ \\
\hline $46-60$ & 20 & 19 & 1 & $5(15)$ & $10(5)$ \\
\hline$>60$ & 17 & 7 & 10 & $6(11)$ & $13(7)$ \\
\hline Gender & & & & & $23(15)$ \\
\hline Female & 44 & 35 & 9 & $10(34)$ & $16(7)$ \\
\hline Male & 28 & 23 & 5 & $11(17)$ & \\
\hline
\end{tabular}

\section{Figures}


A
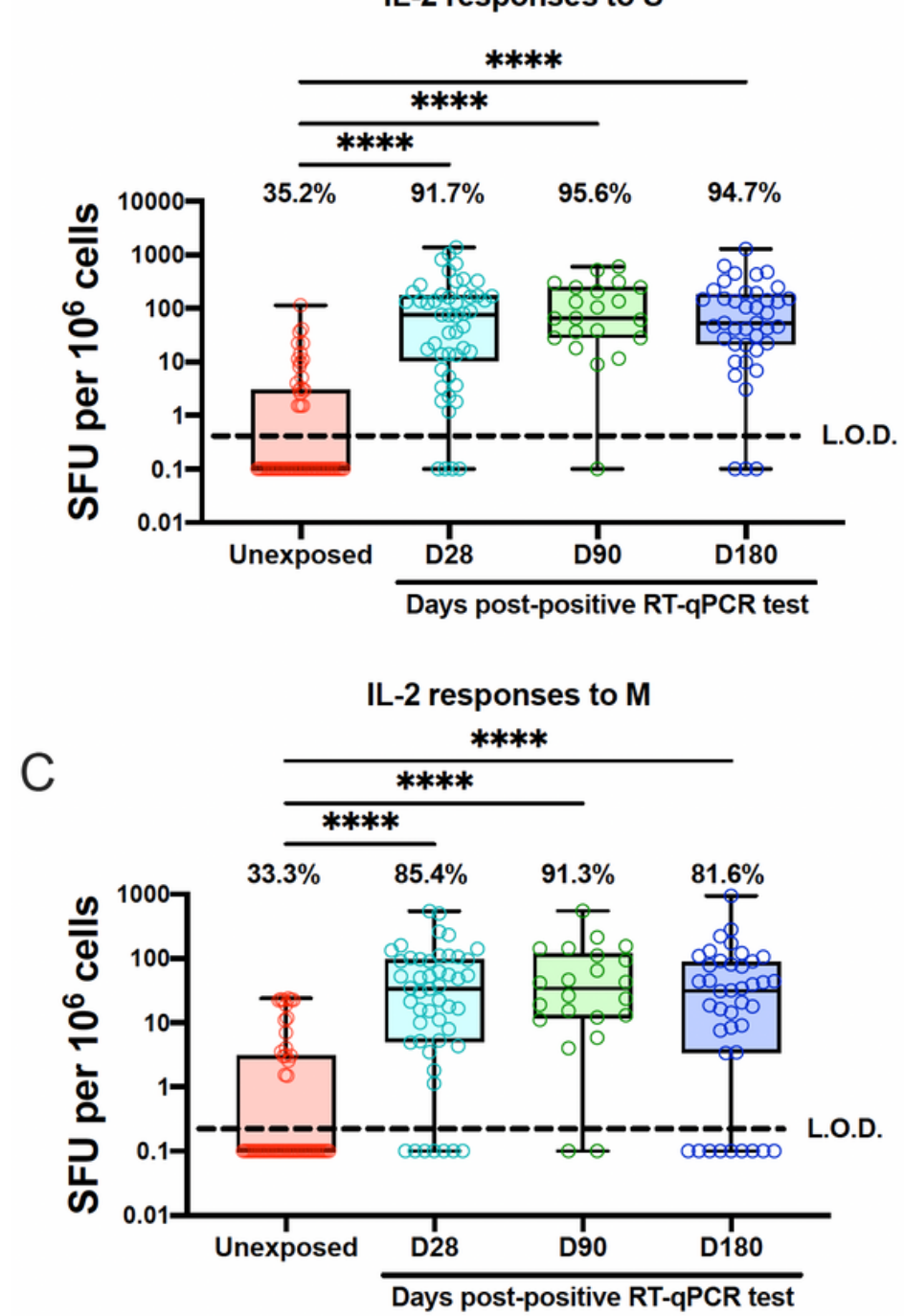

B
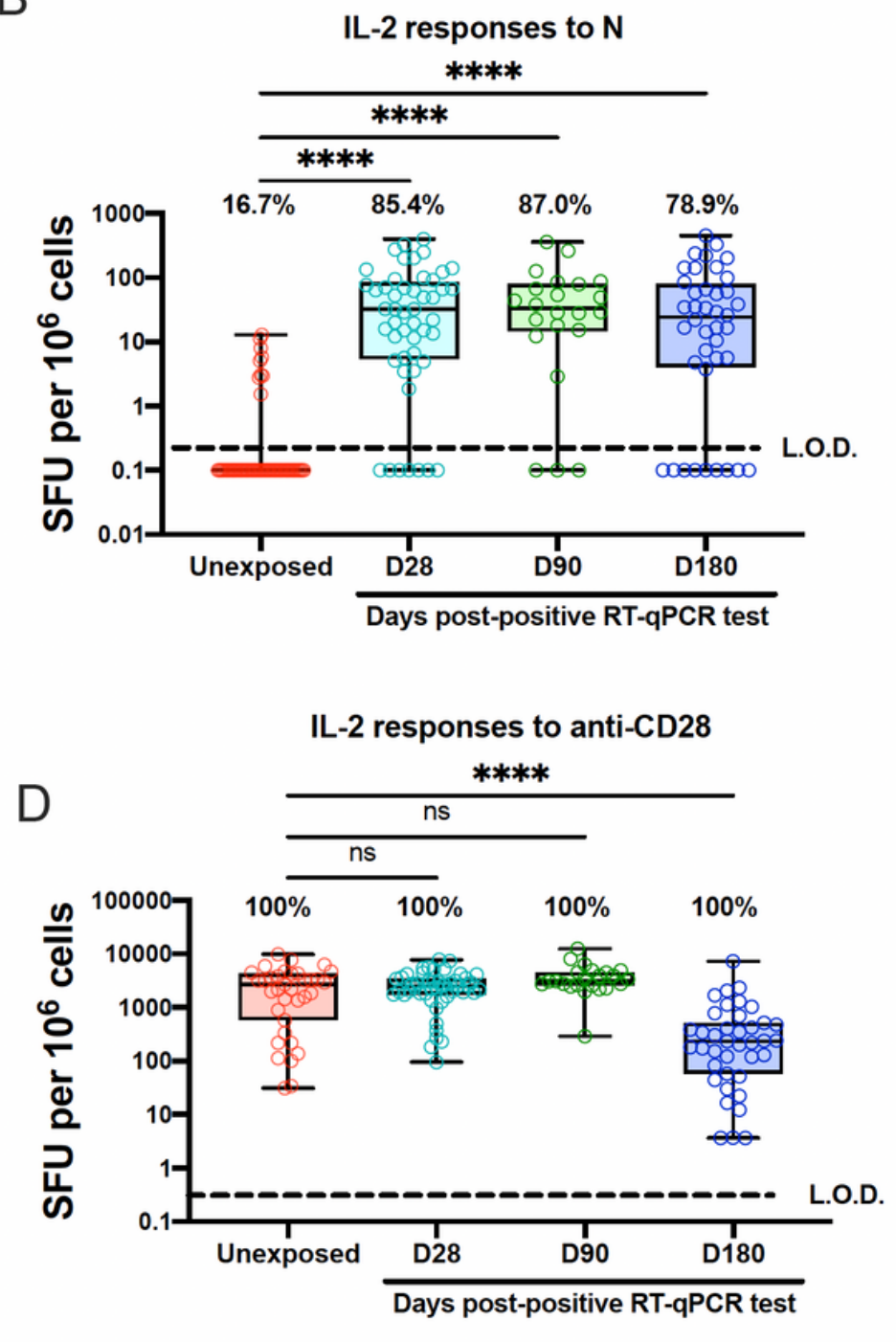

Figure 1

Donors with confirmed SARS-CoV-2 infection show increased IL-2 responses to Spike, nucleocapsid and membrane peptides. PBMCs were isolated from negative control unexposed donors (red), positive-control RT-qPCR-confirmed donors at 28,90 or 180 days post PCR test (cyan, green, blue). These PBMCs were stimulated with spike (A), nucleocapsid (B) or membrane (C) peptides or anti-CD28 as a positive control (d). IL-2 responses were measured by fluorospot assay as spot forming units per million PBMCs. Each condition was run in duplicate and an unstimulated control was subtracted to remove background cytokine production. Zero results are set as 0.1 to allow their inclusion on a log scale. Each condition was run in duplicate and an unstimulated control was subtracted to remove background cytokine production. L.O.D. $=$ limit of detection. Significance calculated by Kruskal-Wallis ANOVA, ${ }^{\star \star \star \star} p<0.001$, n.s. $=$ not significant. 
IFN-y responses to S

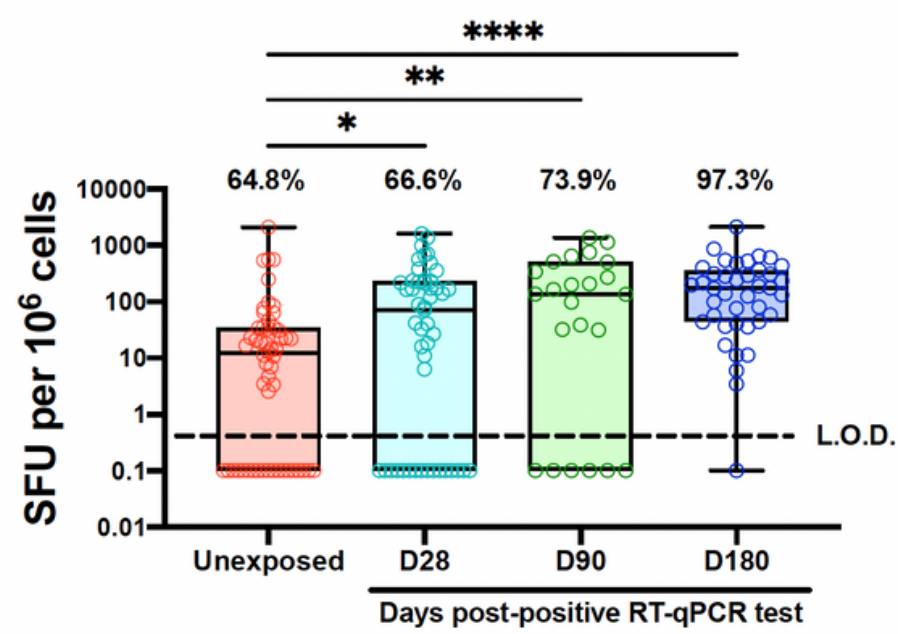

IFN-y responses to $M$

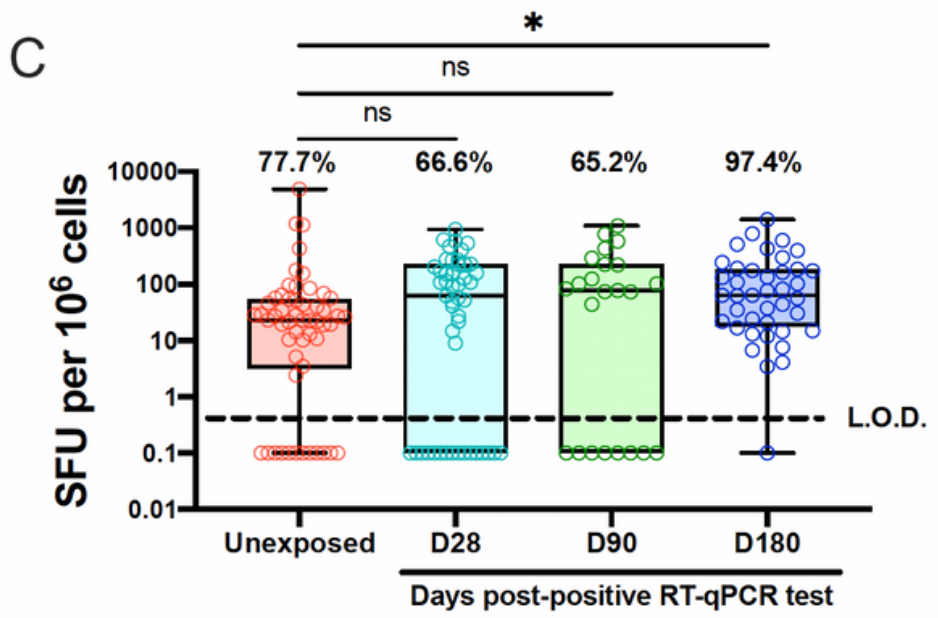

B

IFN-y responses to $\mathrm{N}$

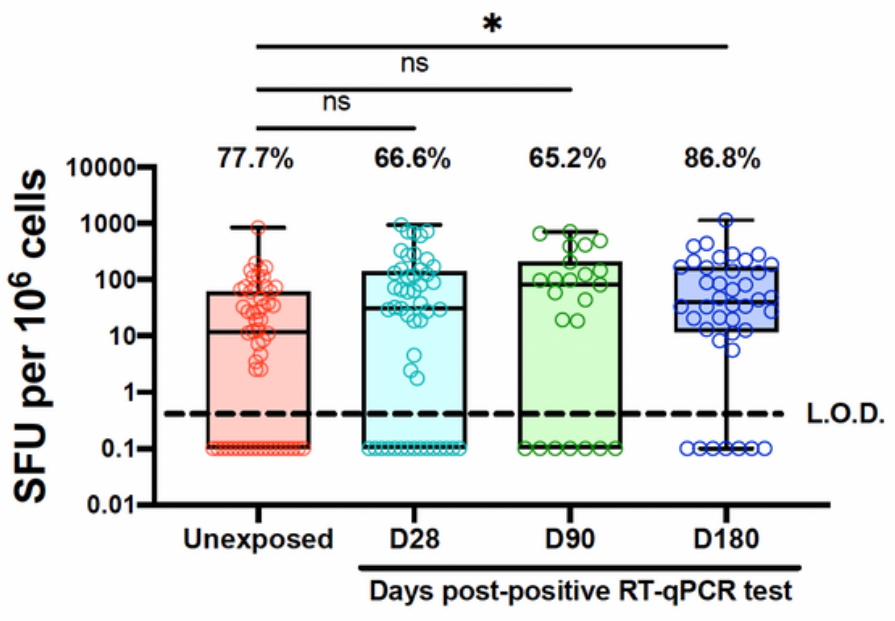

IFN-y responses to CEF

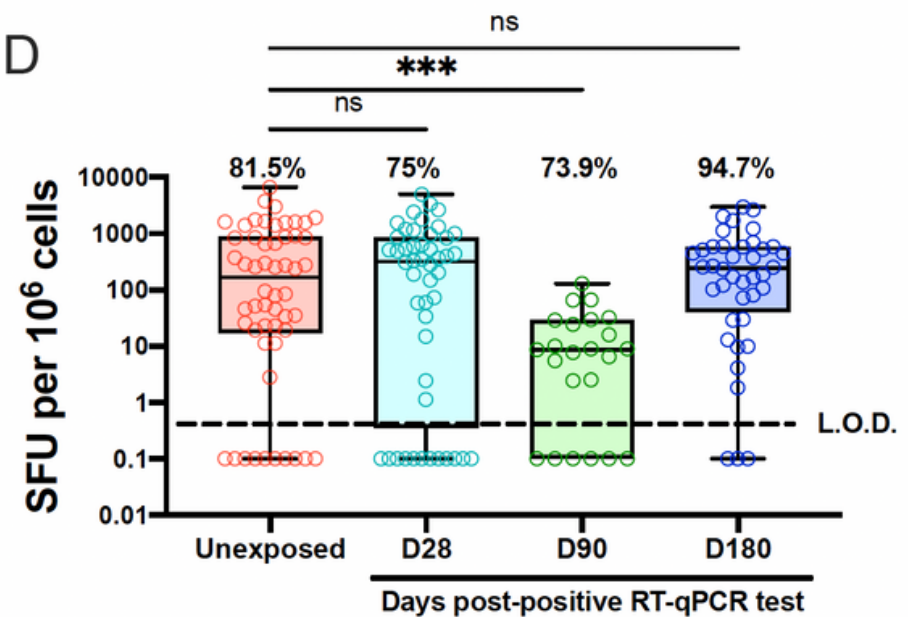

Figure 2

Donors with confirmed SARS-CoV-2 infection show inconsistently higher IFN-y responses to Spike, nucleocapsid and membrane peptides. PBMCs were isolated from negative control unexposed donors (red), positive-control RT-qPCR-confirmed donors at 28,90 or 180 days post PCR test (cyan, green, blue). These PBMCs were stimulated with spike (A), nucleocapsid (B) membrane (C), or positive-control cytomegalovirus/flu/EBV (CEF, d) peptides. IFN-y responses were measured by fluorospot assay as spot forming units per million PBMCs. Each condition was run in duplicate and an unstimulated control was subtracted to remove background cytokine production. Zero results are set as 0.1 to allow their inclusion on a log scale. Each condition was run in duplicate and an unstimulated control was subtracted to remove background cytokine production. L.O.D. = limit of detection. Significance calculated by KruskalWallis ANOVA, ${ }^{*} p<0.05,{ }^{*} p<0.01,{ }^{* \star *} p<0.001,{ }^{* \star \star *} p<0.0001$, n.s. $=$ not significant. 
A

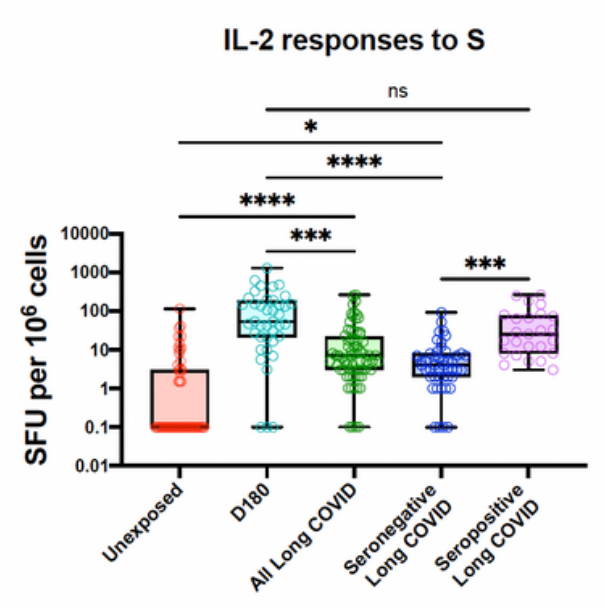

B

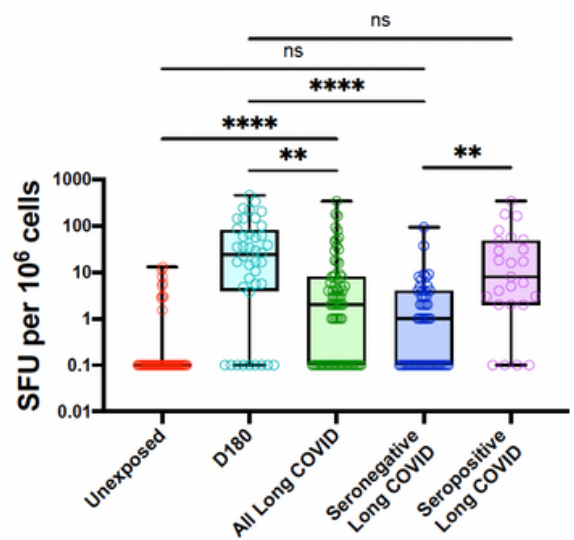

C

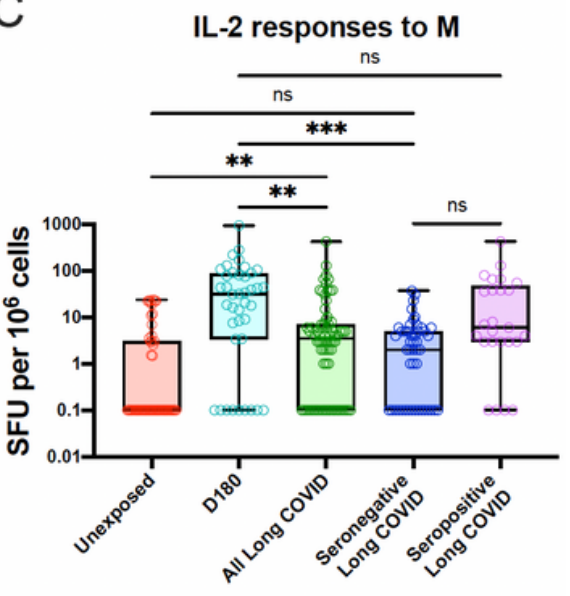

Figure 3

Donors with diagnosed Long COVID show increased IL-2 responses to Spike, nucleocapsid and membrane peptides. PBMCs were isolated from diagnosed Long COVID patients between 6-13 months after symptom onset. These PBMCs were stimulated with spike (A), nucleocapsid (B) or membrane (C) peptides. IL-2 responses were measured by fluorospot assay as spot forming units per million PBMCs. Unexposed (red), and PCR-positive samples at day 180 (cyan) are included from Figure 1. Results either show the entire Long COVID cohort (green) or those stratified by antibody serology as negative (blue) or positive (purple). Zero results are set as 0.1 to allow their inclusion on a log scale. Each condition was run in duplicate and an unstimulated control was subtracted to remove background cytokine production. L.O.D. $=$ limit of detection. Significance calculated by Kruskal-Wallis ANOVA, ${ }^{{ }} p<0.05,{ }^{*} p<<0.01$, $\star \star \star p<0.001, * \star \star \star p<0.0001$, n.s. $=$ not significant. 
A

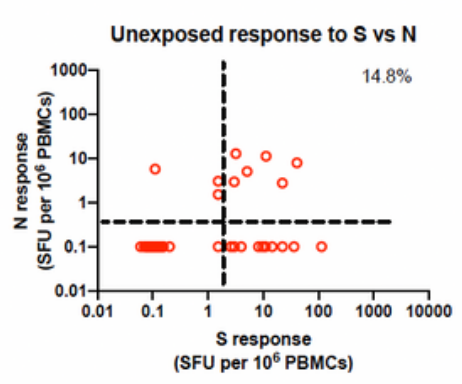

D

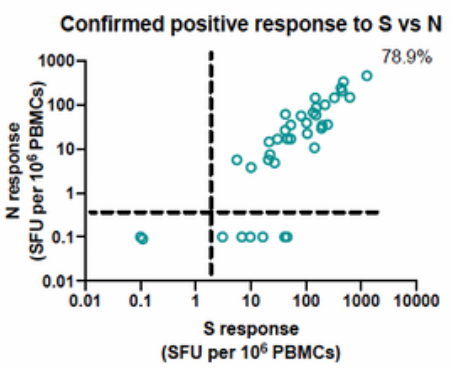

G

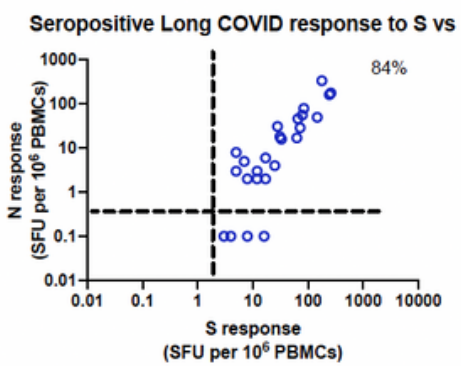

J Seronegative Long COVID response to $\mathrm{S}$ vs $\mathrm{N}$

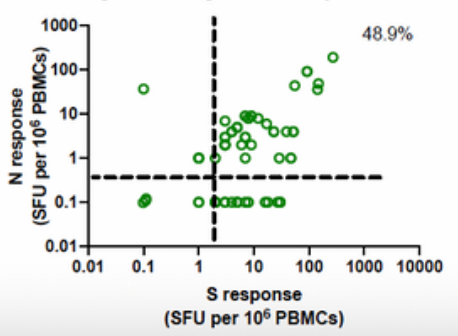

B

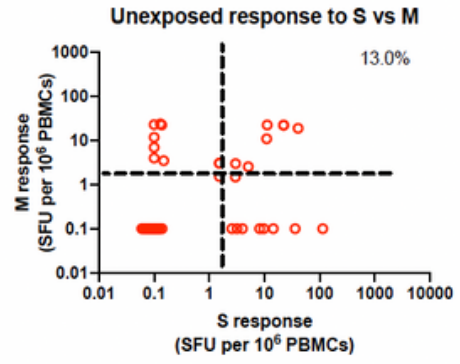

E

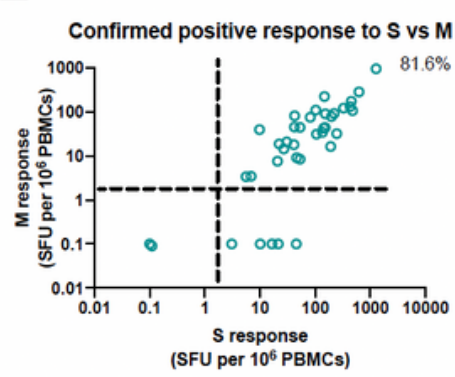

$\mathrm{H}$

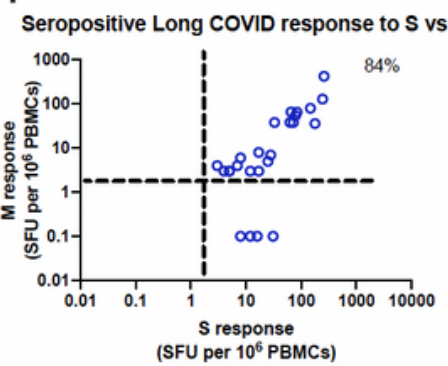

$\mathrm{K}$

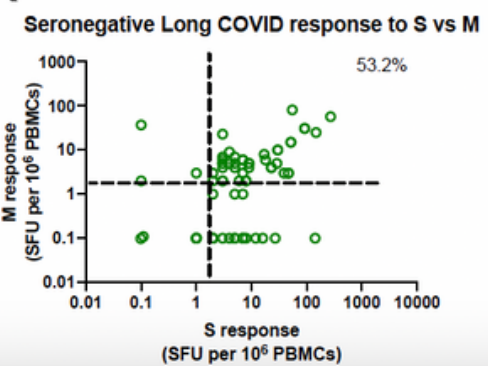

C

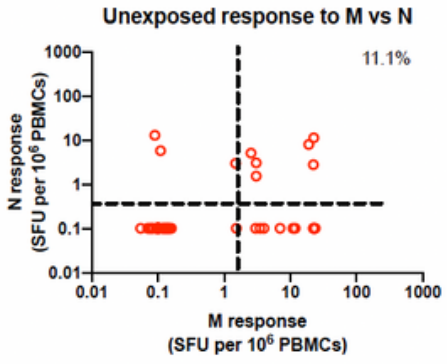

$\mathrm{F}$

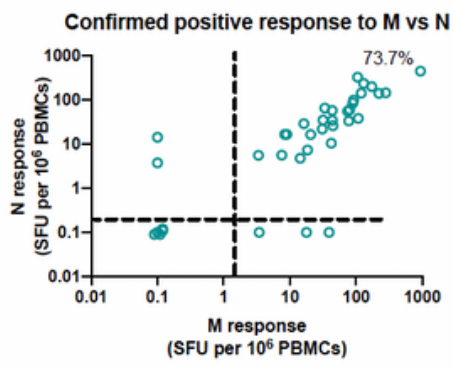

I

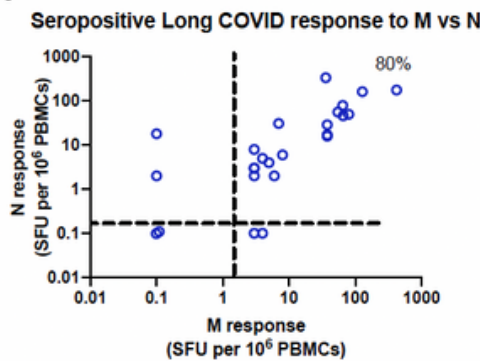

L

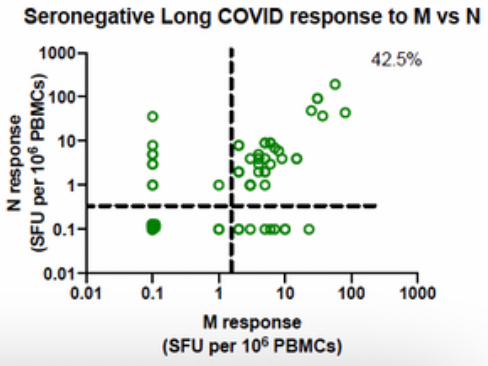

Figure 4

Positive donors are likely to respond to multiple SARS-CoV-2 peptides while unexposed negative control donors do not. Fluorospot results from figure 1 plotted against each other. Dotted lines indicate results higher than the upper quartile for unexposed IL-2 responses to S, N, or M peptides. 


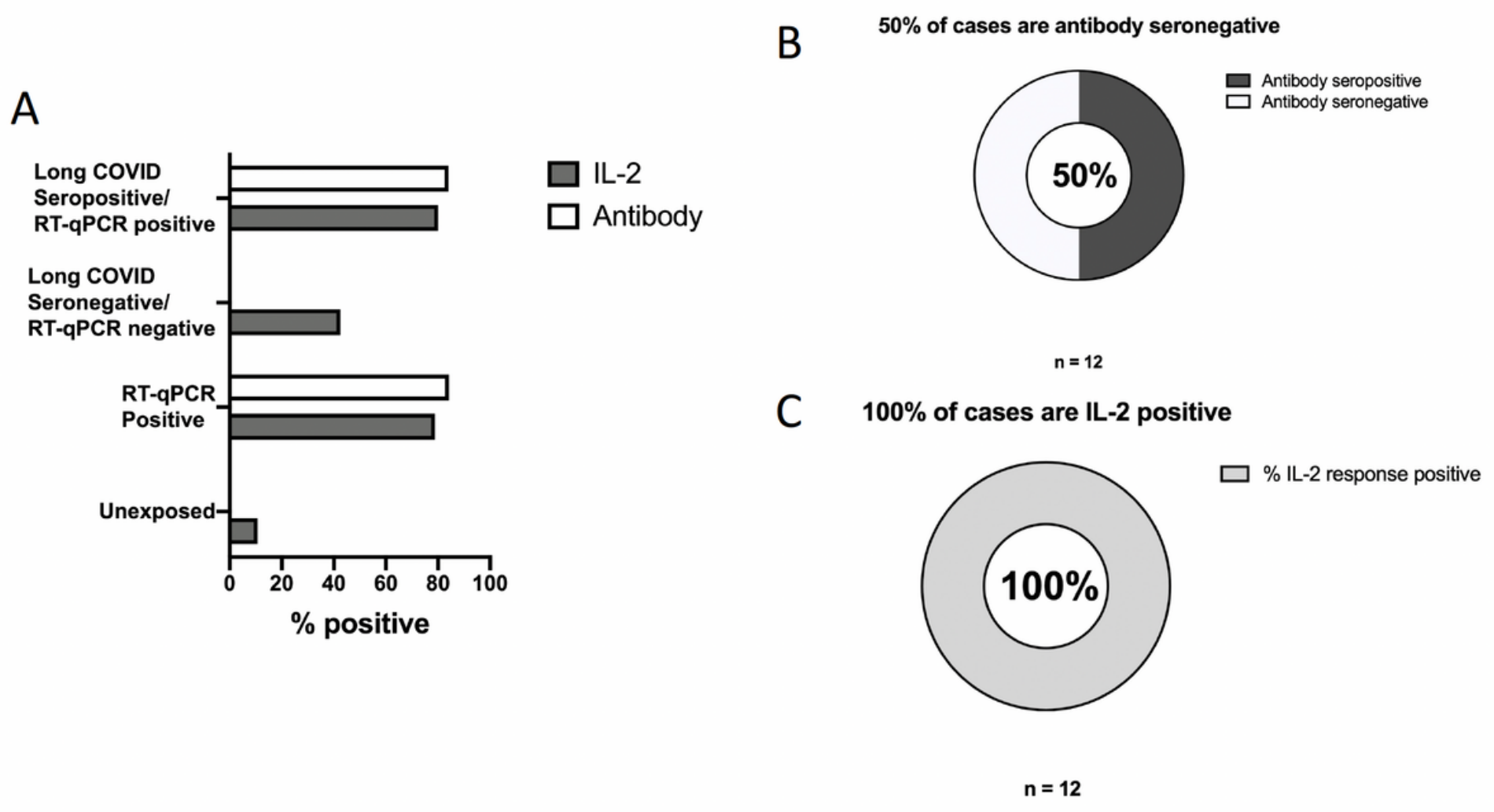

\section{Figure 5}

IL-2 responses to $\mathrm{N}$ and $\mathrm{M}$ peptides are more sensitive to past SARS-CoV-2 infection than antibody serology. A) Percentage of each cohort (unexposed, RT-qPCR positive at D180, Long COVID negative and long COVID positive) who had a positive IL-2 response to $\mathrm{N}$ and $\mathrm{M}$ peptides vs positive antibody responses. B) Percentage of COVID-19 hospitalised patients who were antibody positive for anti-S and anti-N antibodies. C) Percentage of COVID-19 hospitalised patients who were IL-2 positive for $\mathrm{N}$ and $\mathrm{M}$ peptides.

\section{Supplementary Files}

This is a list of supplementary files associated with this preprint. Click to download.

- SupplementaryFigures.pdf 